\title{
ON FALTINGS' ANNIHILATOR THEOREM
}

\author{
TAKESI KAWASAKI
}

(Communicated by Bernd Ulrich)

Dedicated to Professor Shiro Goto on the occasion of his sixtieth birthday

\begin{abstract}
In the present article, the author shows that Faltings' annihilator theorem holds for any Noetherian ring $A$ if $A$ is universally catenary; all the formal fibers of all the localizations of $A$ are Cohen-Macaulay; and the CohenMacaulay locus of each finitely generated $A$-algebra is open.
\end{abstract}

\section{INTRODUCTION}

Throughout the present article, $A$ always denotes a commutative Noetherian ring. We say that the annihilator theorem holds for $A$ if it satisfies the following proposition [4].

The Annihilator Theorem. Let $M$ be a finitely generated $A$-module, $n$ an integer and $Y, Z$ subsets of Spec $A$ which are stable under specialization. Then the following statements are equivalent:

(1) ht $\mathfrak{p} / \mathfrak{q}+\operatorname{depth} M_{\mathfrak{q}} \geq n$ for any $\mathfrak{q} \in \operatorname{Spec} A \backslash Y$ and $\mathfrak{p} \in V(\mathfrak{q}) \cap Z$;

(2) there is an ideal $\mathfrak{b}$ in $A$ such that $V(\mathfrak{b}) \subset Y$ and $\mathfrak{b}$ annihilates the local cohomology modules $H_{Z}^{0}(M), \ldots, H_{Z}^{n-1}(M)$.

Faltings [3] proved that the annihilator theorem holds for $A$ if $A$ has a dualizing complex or if $A$ is a homomorphic image of a regular ring and that (2) always implies (1). Several authors $[1,2,9,10,11]$ tried to extend Faltings' result. In this article, the author shows the following

Theorem 1.1. The annihilator theorem holds for $A$ if

(C1) A is universally catenary;

(C2) all the formal fibers of all the localizations of A are Cohen-Macaulay; and

(C3) the Cohen-Macaulay locus of each finitely generated A-algebra is open.

These conditions are not only sufficient but also necessary for the annihilator theorem. Indeed, Faltings [4] showed that $A$ satisfies (C1)-(C3) whenever the annihilator theorem holds for each $A$-algebra essentially of finite type.

Received by the editors September 15, 2006 and, in revised form, January 11, 2007.

1991 Mathematics Subject Classification. Primary 13D45; Secondary 13C15, 14B15.

Key words and phrases. Annihilators of local cohomologies, Cousin complex, Artin-Rees theorem, Briançon-Skoda theorem.

This work was supported by the Japan Society for the Promotion of Science (the Grant-in-Aid for Scientific Researches (C)(2) 16540032). 
These conditions are also related to the uniform Artin-Rees theorem and the uniform Briançon-Skoda theorem. We give an affirmative answer to the conjecture of Huneke [7, Conjecture 2.13] in the last section.

\section{Preliminaries}

First we recall the definition of the local cohomology functor. A subset $Z$ of $\operatorname{Spec} A$ is said to be stable under specialization if $\mathfrak{p} \in Z$ implies $V(\mathfrak{p}) \subset Z$. Let $M$ be an $A$-module and $Z$ a subset of $\operatorname{Spec} A$ which is stable under specialization. Then we put

$$
H_{Z}^{0}(M)=\{m \in M \mid \operatorname{Supp} A m \subset Z\} .
$$

This is an $A$-submodule of $M$, and $H_{Z}^{0}(-)$ is a left exact functor.

Definition 2.1 ([5, p. 223]). The local cohomology functor $H_{Z}^{p}(-)$ with respect to $Z$ is the right derived functor of $H_{Z}^{0}(-)$.

If $\mathfrak{b}$ is an ideal, then $Z=V(\mathfrak{b})$ is stable under specialization and $H_{Z}^{p}(-)$ coincides with the ordinary local cohomology functor $H_{\mathfrak{b}}^{p}(-)$.

Let $Z$ be a subset of Spec $A$ which is stable under specialization. If $\mathfrak{b}, \mathfrak{b}^{\prime}$ are ideals such that $V(\mathfrak{b}), V\left(\mathfrak{b}^{\prime}\right) \subset Z$, then $V\left(\mathfrak{b} \cap \mathfrak{b}^{\prime}\right) \subset Z$. Therefore the set $\mathcal{F}$ of all ideals $\mathfrak{b}$ such that $V(\mathfrak{b}) \subset Z$ is a directed set with respect to the opposite inclusion. If $\mathfrak{b}$, $\mathfrak{b}^{\prime} \in \mathcal{F}$ are such that $\mathfrak{b}^{\prime} \subset \mathfrak{b}$, then there is a natural transformation $\operatorname{Ext}_{A}^{p}(A / \mathfrak{b},-) \rightarrow$ $\operatorname{Ext}_{A}^{p}\left(A / \mathfrak{b}^{\prime},-\right)$. Since $H_{Z}^{0}(-)=\operatorname{inj} \lim _{\mathfrak{b} \in \mathcal{F}} \operatorname{Hom}(A / \mathfrak{b},-)$, we obtain the natural isomorphism

$$
H_{Z}^{p}(-)=\operatorname{inj} \lim _{\mathfrak{b} \in \mathcal{F}} \operatorname{Ext}_{A}^{p}(A / \mathfrak{b},-) .
$$

The following lemma was essentially given by Raghavan [11, p. 491].

Lemma 2.2. Let $M$ be a finitely generated $A$-module. Then $\mathcal{L}=\left\{H_{Z}^{0}(M) \mid Z \subset\right.$ $\operatorname{Spec} A$ is stable under specialization $\}$ is a finite set.

Proof. Let Ass $M=\left\{\mathfrak{p}_{1}, \ldots, \mathfrak{p}_{r}\right\}$ and $0=M_{1} \cap \cdots \cap M_{r}$ be a primary decomposition of 0 in $M$ where Ass $M / M_{i}=\left\{\mathfrak{p}_{i}\right\}$ for all $i$. Then $H_{Z}^{0}(M)=\bigcup_{V(\mathfrak{b}) \subset Z} 0:_{M} \mathfrak{b}=$ $\bigcap_{\mathfrak{p}_{i} \notin Z} M_{i}$. Therefore $\# \mathcal{L} \leq 2^{r}$.

We need Cousin complexes to prove Theorem 1.1.

Let $M$ be a finitely generated $A$-module. For a prime ideal $\mathfrak{p} \in \operatorname{Supp} M$, the $M$-height of $\mathfrak{p}$ is defined to be $\mathrm{ht}_{M} \mathfrak{p}=\operatorname{dim} M_{\mathfrak{p}}$. If $\mathfrak{b}$ is an ideal in $A$ such that $M \neq \mathfrak{b} M$, let $\operatorname{ht}_{M} \mathfrak{b}=\inf \left\{\operatorname{ht}_{M} \mathfrak{p} \mid \mathfrak{p} \in \operatorname{Supp} M \cap V(\mathfrak{b})\right\}$.

Definition 2.3 ([12]). The Cousin complex $\left(M^{\bullet}, d_{M}^{\bullet}\right)$ of $M$ is defined as follows:

Let $M^{-2}=0, M^{-1}=M$ and $d_{M}^{-2}: M^{-2} \rightarrow M^{-1}$ be the zero map. If $p \geq 0$ and $d_{M}^{p-2}: M^{p-2} \rightarrow M^{p-1}$ is given, then we put

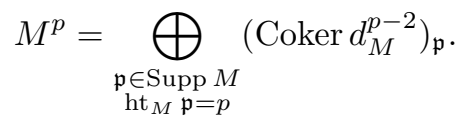

If $\xi \in M^{p-1}$ and $\bar{\xi}$ is the image of $\xi$ in Coker $d_{M}^{p-2}$, then the component of $d_{M}^{p}(\xi)$ in $\left(\text { Coker } d_{M}^{p-2}\right)_{\mathfrak{p}}$ is $\bar{\xi} / 1$.

The following theorem contains [6, Theorems 11.4 and 11.5]. 
Theorem 2.4. Assume that A satisfies (C1)-(C3) and let $M$ be a finitely generated A-module satisfying

(QU) ht $\mathfrak{p} / \mathfrak{q}+\mathrm{ht}_{M} \mathfrak{q}=\mathrm{ht}_{M} \mathfrak{p}$ for any $\mathfrak{p}, \mathfrak{q} \in \operatorname{Supp} M$ such that $\mathfrak{p} \supset \mathfrak{q}$.

Then there is an ideal $\mathfrak{a}$ in A satisfying the following properties:

(1) $V(\mathfrak{a})$ is the non-Cohen-Macaulay locus of $M$. In particular, $\mathrm{ht}_{M} \mathfrak{a}>0$.

(2) Let $Z$ be a subset of $\operatorname{Spec} A$ which is stable under specialization and let $n$ be an integer. If $\mathrm{ht}_{M} \mathfrak{p} \geq n$ for any $\mathfrak{p} \in Z \cap \operatorname{Supp} M$, then $\mathfrak{a} H_{Z}^{p}(M)=0$ for each $p<n$.

(3) Let $x_{1}, \ldots, x_{n} \in A$ be a sequence. If $\mathrm{ht}_{M}\left(x_{1}, \ldots, x_{n}\right) A \geq n$, then $\mathfrak{a}$ annihilates the Koszul cohomology module $H^{p}\left(x_{1}, \ldots, x_{n} ; M\right)$ of $M$ with respect to $x_{1}$, $\ldots, x_{n}$ for any $p<n$.

Proof. Let $M^{\bullet}$ be the Cousin complex of $M$ and $\mathfrak{a}$ the product of all the annihilators of all the non-zero cohomologies of $M^{\bullet}$. Then the ideal $\mathfrak{a}$ is well-defined and satisfies (1). See [8, Corollary 6.4].

We prove (2). Because of (2.1.1), it is enough to show that $\mathfrak{a} \operatorname{Ext}^{p}(A / \mathfrak{b}, M)=0$ for any ideal $\mathfrak{b}$ such that $V(\mathfrak{b}) \subset Z$ and for any $p<n$. Let $\mathfrak{b}$ be such an ideal and let $F_{\bullet}$ be a free resolution of $A / \mathfrak{b}$. The double complex $\operatorname{Hom}\left(F_{\bullet}, M^{\bullet}\right)$ gives two spectral sequences

$$
\begin{aligned}
{ }^{\prime} E_{2}^{p q} & =\operatorname{Ext}^{p}\left(A / \mathfrak{b}, H^{q}\left(M^{\bullet}\right)\right) \Rightarrow H^{p+q}\left(\operatorname{Hom}\left(F_{\bullet}, M^{\bullet}\right)\right), \\
{ }^{\prime} E_{2}^{p q} & =H^{p}\left(\operatorname{Ext}^{q}\left(A / \mathfrak{b}, M^{\bullet}\right)\right) \Rightarrow H^{p+q}\left(\operatorname{Hom}\left(F_{\bullet}, M^{\bullet}\right)\right) .
\end{aligned}
$$

The first spectral sequence tells us that $\mathfrak{a} H^{k}\left(\operatorname{Hom}\left(F_{\bullet}, M^{\bullet}\right)\right)=0$ for any $k$.

On the other hand, " $E_{2}^{p q}=0$ if $p<-1$ or if $q<0$. Let $0 \leq p<n$ be an integer and $\mathfrak{p} \in \operatorname{Supp} M$ such that $\mathrm{ht}_{M} \mathfrak{p}=p$. Since $\mathfrak{b} \not \subset \mathfrak{p}$, we find that $\operatorname{Hom}\left(F_{\bullet},\left(\operatorname{Coker} d_{M}^{p-2}\right)_{\mathfrak{p}}\right)$ is exact. Hence $\operatorname{Hom}\left(F_{\bullet}, M^{p}\right)$ is also exact. Thus ${ }^{\prime \prime} E_{2}^{p q}=0$ if $0 \leq p<n$ and ${ }^{\prime \prime} E_{2}^{-1, q}=\operatorname{Ext}^{q}(A / \mathfrak{b}, M)$. If $k<n$, then ${ }^{\prime \prime} E_{2}^{p, k-p-1}={ }^{\prime \prime} E_{2}^{p, k-p}=0$ whenever $p \neq-1$. Therefore $H^{k-1}\left(\operatorname{Hom}\left(F_{\bullet}, M^{\bullet}\right)\right)={ }^{\prime \prime} E_{2}^{-1, k}=\operatorname{Ext}^{k}(A / \mathfrak{b}, M)$ is annihilated by $\mathfrak{a}$.

Next we consider (3). Let $K \bullet$ be the Koszul complex of $A$ with respect to $x_{1}$, $\ldots, x_{n}$. By considering the double complex $\operatorname{Hom}\left(K_{\bullet}, M^{\bullet}\right)$, instead of $\operatorname{Hom}\left(F_{\bullet}, M^{\bullet}\right)$, we obtain the assertion.

\section{The PRoOf of TheOREM 1.1}

Before the proof of Theorem 1.1, we fix some notation. Let $\mathfrak{X}$ be the free Abelian group with basis $\operatorname{Spec} A$ and let $\mathfrak{X}_{+}=\left\{\sum k_{\mathfrak{p}} \mathfrak{p} \mid k_{\mathfrak{p}} \geq 0\right.$ for all $\left.\mathfrak{p}\right\}$. If $\alpha=k_{1} \mathfrak{p}_{1}+$ $\cdots+k_{n} \mathfrak{p}_{n}$ and $\beta=l_{1} \mathfrak{p}_{1}+\cdots+l_{n} \mathfrak{p}_{n}$ where $\mathfrak{p}_{i} \neq \mathfrak{p}_{j}$ whenever $i \neq j$, then we put

$$
\alpha \vee \beta=\sum_{i=1}^{n} \max \left\{k_{i}, l_{i}\right\} \mathfrak{p}_{i}
$$

It is clear that $(\alpha \vee \beta)+\gamma=(\alpha+\gamma) \vee(\beta+\gamma)$. Let $\alpha=k_{1} \mathfrak{p}_{1}+\cdots+k_{n} \mathfrak{p}_{n} \in \mathfrak{X}_{+}$ and let $Y$ be a subset of $\operatorname{Spec} A$ which is stable under specialization. Then we put $\mathfrak{b}(\alpha, Y)=\prod_{\mathfrak{p}_{i} \in Y} \mathfrak{p}_{i}^{k_{i}}$. Since $V(\mathfrak{b}(\alpha, Y)) \subset Y$, Theorem 1.1 is contained in the following

Theorem 3.1. Assume that A satisfies (C1)-(C3). If $M$ is a finitely generated $A$-module, then there is $\alpha(M) \in \mathfrak{X}_{+}$satisfying the following property: 
Let $Y, Z$ be subsets of $\operatorname{Spec} A$ which are stable under specialization and let $n$ be an integer. If

(A) ht $\mathfrak{p} / \mathfrak{q}+\operatorname{depth} M_{\mathfrak{q}} \geq n$ for any $\mathfrak{q} \in \operatorname{Spec} A \backslash Y$ and $\mathfrak{p} \in V(\mathfrak{q}) \cap Z$, then

(B) $\mathfrak{b}(\alpha(M), Y)$ annihilates $H_{Z}^{0}(M), \ldots, H_{Z}^{n-1}(M)$.

We prove this theorem by Noetherian induction on Supp $M$ and induction on the number of associated primes of $M$.

If $M=0$, then $\alpha(M)=0$ obviously satisfies the assertion. Assume that $M \neq 0$ and that, for any finitely generated $A$-module $M^{\prime}$, there is $\alpha\left(M^{\prime}\right)$ satisfying the assertion of Theorem 3.1 if $\operatorname{Supp} M^{\prime} \subsetneq \operatorname{Supp} M$ or if $\operatorname{Supp} M^{\prime}=\operatorname{Supp} M$ and \# Ass $M^{\prime}<$ \# Ass $M$. We first prove the following claim.

Claim. There is $\alpha^{\prime}(M) \in \mathfrak{X}_{+}$satisfying the following property:

Let $Y, Z$ be subsets of Spec $A$ which are stable under specialization and let $n$ be an integer. If $Y \cap$ Ass $M=\emptyset$ and (A) holds, then (B) holds, too.

Proof. Let Ass $M=\left\{P_{1}, \ldots, P_{r}\right\}$. We may assume that $P_{1} \not \subset P_{2}, \ldots, P_{r}$ without loss of generality. There is an exact sequence

$$
0 \rightarrow L \rightarrow M \rightarrow N \rightarrow 0
$$

such that Ass $L=\left\{P_{2}, \ldots, P_{r}\right\}$ and Ass $N=\left\{P_{1}\right\}$. Since $A$ is universally catenary and $N$ has the unique minimal prime, $N$ satisfies (QU). Let $\mathfrak{a}$ be the ideal obtained by applying Theorem 2.4 to $N$. Then $P_{1} \subsetneq \mathfrak{a}$. Since $P_{1} \not \subset P_{2}, \ldots, P_{r}$, we find that $\mathfrak{a} \not \subset P_{2}, \ldots, P_{r}$. Let $x^{\prime \prime} \in \mathfrak{a} \backslash\left(P_{1} \cup \cdots \cup P_{r}\right)$.

Since $\operatorname{Supp} L \subsetneq \operatorname{Supp} M$ or since $\operatorname{Supp} L=\operatorname{Supp} M$ and \# Ass $L<\# \operatorname{Ass} M$, there is $\alpha(L) \in \mathfrak{X}_{+}$satisfying the assertion of Theorem 3.1. Let $\alpha(L)=k_{1} Q_{1}+$ $\cdots+k_{s} Q_{s}$. We may assume that $Q_{1}, \ldots, Q_{s_{0}} \not \subset P_{1} \cup \cdots \cup P_{r}$ and $Q_{s_{0}+1}, \ldots$, $Q_{s} \subset P_{1} \cup \cdots \cup P_{r}$. Let $x^{\prime} \in Q_{1}^{k_{1}} \cdots Q_{s_{0}}^{k_{s_{0}}} \backslash P_{1} \cup \cdots \cup P_{r}$ and $x=x^{\prime} x^{\prime \prime}$.

Since $x$ is an $M$-non-zero divisor, $\operatorname{Supp} M / x M \subsetneq \operatorname{Supp} M$. We want to show that $\alpha^{\prime}(M)=\alpha(M / x M)$ satisfies the assertion of the claim.

Let $Y, Z$ be subsets of $\operatorname{Spec} A$ which are stable under specialization and let $n$ be an integer. Assume that $Y \cap \operatorname{Ass} M=\emptyset$ and ht $\mathfrak{p} / \mathfrak{q}+\operatorname{depth} M_{\mathfrak{q}} \geq n$ for any $\mathfrak{q} \in \operatorname{Spec} A \backslash Y$ and $\mathfrak{p} \in V(\mathfrak{q}) \cap Z$. If $\mathfrak{p} \in Z \cap \operatorname{Supp} N$, then ht $\mathfrak{p} / P_{1}+\operatorname{depth} M_{P_{1}} \geq n$ because $\operatorname{Supp} N=V\left(P_{1}\right)$ and $P_{1} \notin Y$. Since depth $M_{P_{1}}=0$, we have

$$
\operatorname{ht}_{N} \mathfrak{p}=\operatorname{ht} \mathfrak{p} / P_{1} \geq n \quad \text { for any } \mathfrak{p} \in Z \cap \operatorname{Supp} N .
$$

By using Theorem $2.4(2)$, we find that $x^{\prime \prime} H_{Z}^{p}(N)=0$ for any $p<n$.

Let $\mathfrak{q} \in \operatorname{Spec} A \backslash\left(Y \cup V\left(x^{\prime \prime} A\right)\right)$ and $\mathfrak{p} \in V(\mathfrak{q}) \cap Z$. Since $x^{\prime \prime} \notin \mathfrak{q}, N_{\mathfrak{q}}$ is CohenMacaulay. If $N_{\mathfrak{q}} \neq 0$, then $\mathfrak{p} \in Z \cap \operatorname{Supp} N$ and hence

$$
\begin{aligned}
\operatorname{ht} \mathfrak{p} / \mathfrak{q}+\operatorname{depth} N_{\mathfrak{q}} & =\operatorname{ht} \mathfrak{p} / \mathfrak{q}+\operatorname{dim} N_{\mathfrak{q}} \\
& =\operatorname{ht}_{N} \mathfrak{p} \geq n .
\end{aligned}
$$

Here we used (3.1.1). If $N_{\mathfrak{q}}=0$, then $\operatorname{depth} N_{\mathfrak{q}}=\infty$ and hence ht $\mathfrak{p} / \mathfrak{q}+\operatorname{depth} N_{\mathfrak{q}} \geq$ $n$. Since $\mathfrak{q} \notin Y$, the assumption tells us that ht $\mathfrak{p} / \mathfrak{q}+\operatorname{depth} M_{\mathfrak{q}} \geq n$. Therefore ht $\mathfrak{p} / \mathfrak{q}+\operatorname{depth} L_{\mathfrak{q}} \geq n$. Because of the induction hypothesis,

$$
\mathfrak{b}\left(\alpha(L), Y \cup V\left(x^{\prime \prime} A\right)\right) H_{Z}^{p}(L)=0
$$

for $p<n$. 
Since $x^{\prime \prime} \notin P_{1} \cup \cdots \cup P_{r}, P_{1}, \ldots, P_{r} \notin Y$ and $Q_{s_{0}+1}, \ldots, Q_{s} \subset P_{1} \cup \ldots \cup P_{r}$, we have $Q_{s_{0}+1}, \ldots, Q_{s} \notin Y \cup V\left(x^{\prime \prime} A\right)$. Therefore $x^{\prime} \in Q_{1}^{k_{1}} \cdots Q_{s_{0}}^{k_{s_{0}}} \subset \mathfrak{b}\left(\alpha(L), Y \cup V\left(x^{\prime \prime} A\right)\right)$ and hence $x^{\prime} H_{Z}^{p}(L)=0$ if $p<n$. Since $H_{Z}^{p}(L) \rightarrow H_{Z}^{p}(M) \rightarrow H_{Z}^{p}(N)$ is exact, $x H_{Z}^{p}(M)=0$ if $p<n$.

Since $x$ is an $M$-non-zero divisor, $H_{Z}^{0}(M)=0$ and

$$
0 \rightarrow H_{Z}^{p-1}(M) \rightarrow H_{Z}^{p-1}(M / x M) \rightarrow H_{Z}^{p}(M) \rightarrow 0
$$

is exact for $p<n$ and ht $\mathfrak{p} / \mathfrak{q}+\operatorname{depth}(M / x M)_{\mathfrak{q}} \geq n-1$ for any $\mathfrak{q} \in \operatorname{Spec} A \backslash Y$ and any $\mathfrak{p} \in V(\mathfrak{q}) \cap Z$. Therefore $\mathfrak{b}\left(\alpha^{\prime}(M), Y\right)=\mathfrak{b}(\alpha(M / x M), Y)$ annihilates $H_{Z}^{p}(M)$ if $p<n$.

Next we construct $\alpha(M)$. Let Ass $M=\left\{P_{1}, \ldots, P_{r}\right\}$ and $0=M_{1} \cap \cdots \cap M_{r}$ be a primary decomposition of 0 in $M$ such that Ass $M / M_{i}=\left\{P_{i}\right\}$. Then there are integers $k_{1}, \ldots, k_{r}$ such that $P_{i}^{k_{i}} M \subset M_{i}$ for each $i$.

Let $\left\{H_{Z}^{0}(M) \mid Y \subset \operatorname{Spec} A\right.$ is stable under specialization $\}=\left\{L_{1}, \ldots, L_{s}\right\}$. Assume that $L_{1}=0$ and $L_{2}, \ldots, L_{s} \neq 0$. Since $\operatorname{Supp} M / L_{i} \subsetneq \operatorname{Supp} M$ or $\operatorname{Supp} M / L_{i}=\operatorname{Supp} M, \# \operatorname{Ass} M / L_{i}<\# \operatorname{Ass} M$, there is $\alpha\left(M / L_{i}\right) \in \mathfrak{X}_{+}$satisfying the assertion of Theorem 3.1 for each $i=2, \ldots, s$. We put $\alpha(M)=$ $\alpha^{\prime}(M) \vee\left[\sum k_{i} P_{i}+\alpha\left(M / L_{2}\right) \vee \cdots \vee \alpha\left(M / L_{s}\right)\right]$. Then $\alpha(M)$ has the required property.

Indeed, let $Y, Z$ be subsets of Spec $A$ which are stable under specialization and let $n$ be an integer. If $H_{Y}^{0}(M)=0$, then $Y \cap$ Ass $M=\emptyset$ and hence $\mathfrak{b}\left(\alpha^{\prime}(M), Y\right)$ annihilates $H_{Z}^{0}(M), \ldots, H_{Z}^{n-1}(M)$. Assume that $H_{Y}^{0}(M)=L_{j}$ for some $2 \leq$ $j \leq s$. If $\mathfrak{q} \in \operatorname{Spec} A \backslash Y$ and $\mathfrak{p} \in V(\mathfrak{q}) \cap Z$, then $\left(L_{j}\right)_{\mathfrak{q}}=0$ and hence ht $\mathfrak{p} / \mathfrak{q}+$ $\operatorname{depth}\left(M / L_{j}\right)_{\mathfrak{q}}=\operatorname{ht} \mathfrak{p} / \mathfrak{q}+\operatorname{depth} M_{\mathfrak{q}} \geq n$. Therefore $\mathfrak{b}\left(\alpha\left(M / L_{j}\right), Y\right)$ annihilates $H_{Z}^{0}\left(M / L_{j}\right), \ldots, H_{Z}^{n-1}\left(M / L_{j}\right)$. On the other hand, since there is a monomorphism

$$
L_{j}=\bigcap_{P_{i} \notin Y} M_{i} \hookrightarrow \bigoplus_{P_{i} \in Y} M / M_{i},
$$

we find that $\mathfrak{b}\left(\sum k_{i} P_{i}, Y\right) L_{j}=0$. Since $H_{Z}^{p}\left(L_{j}\right) \rightarrow H_{Z}^{p}(M) \rightarrow H_{Z}^{p}\left(M / L_{j}\right)$ is exact, $\mathfrak{b}\left(\sum k_{i} P_{i}+\alpha\left(M / L_{j}\right), Y\right)$ annihilates $H_{Z}^{0}(M), \ldots, H_{Z}^{n-1}(M)$. Thus (B) holds.

If $L_{1}, \ldots, L_{s}$ are all non-zero, we put $\alpha(M)=\sum k_{i} P_{i}+\alpha\left(M / L_{1}\right) \vee \cdots \vee \alpha\left(M / L_{s}\right)$. We can show that $\alpha(M)$ satisfies the assertion of Theorem 3.1 in the same way as above. The proof of Theorem 1.1 is completed.

The following corollary is an improvement of [11, Theorem 3.1].

Corollary 3.2. Assume that A satisfies (C1)-(C3). If $M$ is a finitely generated $A$-module, then there is a positive integer $k$ satisfying the following property:

Let $\mathfrak{a}, \mathfrak{b}$ be ideals in $A$ and let $n$ be an integer. If $\mathrm{ht} \mathfrak{p} / \mathfrak{q}+\operatorname{depth} M_{\mathfrak{q}} \geq n$ for any $\mathfrak{q} \in \operatorname{Spec} A \backslash V(\mathfrak{b})$ and $\mathfrak{p} \in V(\mathfrak{a}+\mathfrak{q})$, then $\mathfrak{b}^{k} H_{\mathfrak{a}}^{p}(M)=0$ for all $p<n$.

Proof. Let $\alpha(M)=k_{1} \mathfrak{p}_{1}+\cdots+k_{r} \mathfrak{p}_{r}$ and $k=k_{1}+\cdots+k_{r}$. Then $\mathfrak{b}(\alpha(M), V(\mathfrak{b})) \supset$ $\mathfrak{b}^{k}$.

\section{A conjecture of Huneke}

The following theorem is an affirmative answer to Conjecture 2.13 of [7]. Its proof is similar to that of Theorem 2.4.

Theorem 4.1. Assume that A satisfies (C1)-(C3) and let $M$ be a finitely generated A-module satifying (QU). Then there is an ideal $\mathfrak{a}$ in $A$ which satisfies the following requirements: 
(1) $\mathrm{ht}_{M} \mathfrak{a}>0$.

(2) If

$0 \longrightarrow F^{-n} \stackrel{f^{-n}}{\longrightarrow} F^{-n+1} \longrightarrow \cdots \longrightarrow F^{-1} \stackrel{f^{-1}}{\longrightarrow} F^{0}$

is any complex of finitely generated free A-modules such that

(a) $\operatorname{rank} f^{-n}=\operatorname{rank} F^{-n}$,

(b) $\operatorname{rank} F^{i}=\operatorname{rank} f^{i}+\operatorname{rank} f^{i-1}$ for each $-n<i<0$,

(c) $\operatorname{ht}_{M} I_{r_{i}}\left(f^{i}\right) \geq-i$ for each $-n \leq i<0$ where $r_{i}=\operatorname{rank} f_{i}$ for each $i$,

then $\mathfrak{a} H^{p}\left(F^{\bullet} \otimes M\right)=0$ for all $p<0$. Here $I_{r_{i}}\left(f^{i}\right)$ denotes the ideal generated by all the $r_{i}$-minors of the representation matrix of $f^{i}$.

Proof. Let $M^{\bullet}$ be the Cousin complex of $M$ and let $\mathfrak{a}$ be the product of all the annihilators of all the non-zero cohomologies of $M^{\bullet}$. Then $\mathfrak{a}$ satisfies (1). The double complex $F^{\bullet} \otimes M^{\bullet}$ gives a spectral sequence

$$
{ }^{\prime} E_{2}^{p q}=H^{p}\left(F^{\bullet} \otimes H^{q}\left(M^{\bullet}\right)\right) \Rightarrow H^{p+q}\left(F^{\bullet} \otimes M^{\bullet}\right),
$$

which tells us that $\mathfrak{a} H^{p}\left(F^{\bullet} \otimes M^{\bullet}\right)=0$ for all $p$. On the other hand, $F^{\bullet} \otimes M^{\bullet}$ gives another spectral sequence " $E_{2}^{p q} \Rightarrow H^{p+q}\left(F^{\bullet} \otimes M^{\bullet}\right)$ where ${ }^{\prime \prime} E_{2}^{p q}$ is the cohomology of

$$
H^{q}\left(F^{\bullet} \otimes M^{p-1}\right) \rightarrow H^{q}\left(F^{\bullet} \otimes M^{p}\right) \rightarrow H^{q}\left(F^{\bullet} \otimes M^{p+1}\right) .
$$

If $0 \leq p<n$ and $\mathfrak{p} \in \operatorname{Supp} M$ such that $p=\mathrm{ht}_{M} \mathfrak{p}$, then

$$
0 \longrightarrow\left(F^{-n}\right)_{\mathfrak{p}} \longrightarrow \cdots \longrightarrow\left(F^{-p}\right)_{\mathfrak{p}}
$$

is split exact and hence $H^{q}\left(F^{\bullet} \otimes M^{p}\right)=0$ if $q<-p$. Therefore " $E_{2}^{p q}=0$ if $p>0$ and $p+q<0$. Furthermore ${ }^{\prime \prime} E_{2}^{-1, q}=H^{q}\left(F^{\bullet} \otimes M\right)$ for each $q<0$. Of course, ${ }^{\prime \prime} E_{2}^{p q}=0$ if $p<-1$. Thus $H^{p}\left(F^{\bullet} \otimes M\right)={ }^{\prime \prime} E_{2}^{-1, p}=H^{p-1}\left(F^{\bullet} \otimes M^{\bullet}\right)$ is annihilated by $\mathfrak{a}$ if $p<0$.

\section{REFERENCES}

[1] M. Brodmann, Ch. Rotthaus, and R. Y. Sharp, On annihilators and associated primes of local cohomology modules, J. Pure Appl. Algebra 153 (2000), no. 3, 197-227. MR2002b:13027

[2] M. P. Brodmann and R. Y. Sharp, Local cohomology: an algebraic introduction with geometric applications, Cambridge Studies in Advanced Mathematics, vol. 60, Cambridge University Press, Cambridge, 1998. MR99h:13020

[3] Gerd Faltings, Über die Annulatoren lokaler Kohomologiegruppen, Arch. Math. (Basel) 30 (1978), no. 5, 473-476. MR58:22058

[4] , Der Endlichkeitssatz in der lokalen Kohomologie, Math. Ann. 255 (1981), no. 1, 45-56. MR82f:13003

[5] Robin Hartshorne, Residues and duality, Lecture notes of a seminar on the work of A. Grothendieck, given at Harvard 1963/64. With an appendix by P. Deligne. Lecture Notes in Mathematics, No. 20, Springer-Verlag, Berlin, 1966. MR36:5145

[6] Melvin Hochster and Craig Huneke, Tight closure, invariant theory, and the Briançon-Skoda theorem, J. Amer. Math. Soc. 3 (1990), no. 1, 31-116. MR91g:13010

[7] Craig Huneke, Uniform bounds in Noetherian rings, Invent. Math. 107 (1992), no. 1, 203223. MR93b:13027

[8] Takesi Kawasaki, Finiteness of Cousin cohomologies, to appear in Trans. Amer. Math. Soc.

[9] K. Khashyarmanesh and Sh. Salarian, Faltings' theorem for the annihilation of local cohomology modules over a Gorenstein ring, Proc. Amer. Math. Soc. 132 (2004), no. 8, 2215-2220 (electronic). MR2052396 (2005f:13021)

[10] _ Uniform annihilation of local cohomology modules over a Gorenstein ring, Comm. Algebra 34 (2006), no. 5, 1625-1630. MR2229481

[11] K. Raghavan, Uniform annihilation of local cohomology and of Koszul homology, Math. Proc. Cambridge Philos. Soc. 112 (1992), no. 3, 487-494. MR94e:13033 
[12] Rodney Y. Sharp, The Cousin complex for a module over a commutative Noetherian ring, Math. Z. 112 (1969), 340-356. MR41:8400

Department of Mathematics and Information Sciences, Tokyo Metropolitan UniverSity, Minami-Ohsawa 1-1, HaChiOJi, TOKYo 192-0397, JAPAN

E-mail address: kawasaki@tmu.ac.jp 